\title{
Dispersion and Sensitivity Analysis of Quasi-Scholte Wave Liquid Sensing by Analytical Methods
}

\author{
Onursal Önen \\ Aselsan Defense Industries, Mehmet Akif Ersoy Mh., 296. Cad. No. 16, Yenimahalle, 06370 Ankara, Turkey \\ Correspondence should be addressed to Onursal Önen; oonen@aselsan.com.tr
}

Received 30 June 2017; Revised 15 October 2017; Accepted 17 October 2017; Published 7 December 2017

Academic Editor: Christos Riziotis

Copyright ( 2017 Onursal Önen. This is an open access article distributed under the Creative Commons Attribution License, which permits unrestricted use, distribution, and reproduction in any medium, provided the original work is properly cited.

\begin{abstract}
Ultrasonic-guided wave sensing relies on perturbation of wave propagation by changing physical properties of the target media. Solid waveguides, through which guided waves can be transduced between the transducer and the target media, are frequently employed for liquid sensing and several other applications. In this manuscript, liquid sensing sensitivity of dispersive quasi-Scholte waves, which are guided interface waves that travel at the solid-liquid boundary, is investigated. Dispersion analysis of quasi-Scholte waves is done and sensitivities of quasi-Scholte waves to changes in fluid density and speed of sound in a dipstick configuration are analyzed. An experimentally verified analytical model based on a global matrix approach is employed in a nondimensional manner to generate representative dispersion and sensitivity surfaces. Optimum configurations with respect to the material properties of the liquid and of the waveguide are illustrated, which would enable optimal quasi-Scholte liquid sensing.
\end{abstract}

\section{Introduction}

Ultrasonic sensors have become popular in the recent decades in a wide range of applications for research purposes and as commercial products such as displacement sensors [1], in liquid property measurements [2-11], cure monitoring [12], material property determination $[13,14]$, filling level sensor [15], temperature sensing [16-19], and structural health monitoring [20]. Ultrasonic sensing relies on monitoring quantitative changes in ultrasonic wave propagation, usually in terms of ultrasonic wave velocity or attenuation resulting from perturbations in target sensing media/structure. Ultrasonic wave propagation can be perturbed by several parameters such as changing temperature, mechanical stress, stiffness, boundary conditions, density, polymerization, bulk and shear viscosity, and state. Through careful selection and design of transduction mechanism, these changes can be isolated from each other, enabling sensing of target quantity. By means of transduction mechanism, ultrasonic sensing techniques can be categorized as singleand multitransducer systems. In a single transducer method, the same transducer is employed for transmit/receive operation, whereas in multitransducer systems, at least one (or more) transducer(s) are used to transmit waves and one or more transducers are used for receiving the signal.

Frequently, waveguides that are used to transmit ultrasonic wave between the target media/structure and the transducer are employed in ultrasonic sensing systems. They are essential in applications, where target physical conditions are unsuitable for direct transduction (unconventional geometry, liquid state, extreme temperatures, and high radioactivity), or inaccessible (buried, encapsulated). As can be depicted from the literature, there is no single solution to guided ultrasonic sensing with several different wave types, waveguide structure and material, and electrical periphery. Each ultrasonic sensor problem requires extensive examination of the problem, careful selection of transducer, waveguide, and electrical periphery type, specifically for that problem.

Scholte wave ultrasonic sensing is one of the promising methods, which employs Scholte interface waves for characterization and sensing of liquids. Scholte waves are elastic waves that propagate at the solid-liquid interface. They are nondispersive for acoustically thick interfacing media; and 
dispersive propagation can be observed, for example, when an acoustically thin (i.e., for small thickness-frequency products) solid waveguide is immersed in a liquid. Dispersive Scholte waves, named as quasi-Scholte mode [3], have varying phase and group velocity as a function of frequency. The energy content of the waves is concentrated in a few wavelengths around the interface in each medium with its distribution depending on the material properties, thickness, and frequency at quasi-Scholte mode. Scholte wave velocity is always lower than the longitudinal velocity of the fluid and the transverse velocity of the interfacing solids [21]. Sensing applications with quasi-Scholte waves have been reported for liquid characterization $[3,11,22-24]$ with their most important advantages being single transducer compatibility, high sensitivity to perturbations in liquids, and lossless transmission through the waveguide enabling separation of transducer and the target. Dipstick sensing system can be employed, which briefly consists of a thin plate solid waveguide, to which a shear wave transducer is attached to one end, and the other side is immersed in the target liquid. A waveform generator feeds the transducer with pulses to generate Lamb waves in the dry end of the waveguide, which is converted to Scholte waves upon entry to the liquidimmersed side. Scholte waves are reflected back from the end of the waveguide, which is reconverted to Lamb waves while passing through the wet/dry boundary and then received back by the transducer. The received signal is postprocessed to extract Scholte wave velocity that is used to characterize the fluid. Interested readers may refer to $[2,3,22]$ for further details on the experimental setup and postprocessing.

In this manuscript, dispersion analysis of quasi-Scholte waves and sensitivities of quasi-Scholte and Scholte modes to changes in fluid density and speed of sound in a dipstick configurations are presented. An analytical model, which is described in the next section, is used to investigate dispersion and sensitivity of quasi-Scholte waves with respect to material properties of the liquid and of the waveguide.

\section{Analytical Modelling}

Quasi-Scholte wave dispersion and sensitivity are analytically investigated in a multilayer global matrix approach following a convention similar to Lowe's [25] based on Thomson Haskell approach [26], with the main difference being using partial wave vectors instead of layer matrices. The partial waves, positive and negative travelling longitudinal and shear waves, are defined to trace guided wave propagation $[2,25,27-32]$. Detailed derivation of the stress and displacement expressions can also be found in [25]. A brief overview of the analytical model is presented below. Starting with Navier-Stokes equation (1), Helmholtz decomposition is applied for the displacement field $(u)$, assuming longitudinal (represented by scalar field $\Phi$ ) and shear (represented by vector field $\Psi$ ) wave propagation is not coupled with each other (2). In (1), $\rho$ is the density; $\lambda$ and $\mu$ are the Lame constants.

$$
\rho \frac{\partial^{2} u}{\partial t^{2}}=(\lambda+\mu) \nabla(\nabla \cdot u)+\mu \nabla^{2} u
$$

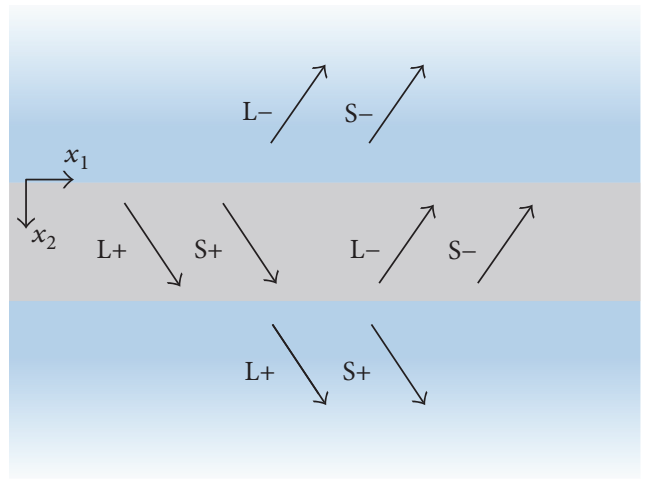

Figure 1: An overview of the multilayer system.

$$
\begin{aligned}
u & =u_{(S)}+u_{(L)} \\
& =\nabla \phi+\nabla \times \psi, \\
\phi & =A_{(L)} e^{i(k \cdot x-\omega t)} \\
& =A_{(L)} e^{i\left(k_{1} x_{1}+k_{2} x_{2}+k_{3} x_{3}-\omega t\right)}, \\
\psi & =A_{(S)} e^{i(k \cdot x-\omega t)} \\
& =A_{(S)} e^{i\left(k_{1} x_{1}+k_{2} x_{2}+k_{3} x_{3}-\omega t\right)} .
\end{aligned}
$$

Using harmonic wave assumption, the equations for the two wave fields ((3) and (4)) are solved and expressions for the displacements and stresses are obtained by the theory of elasticity equations (also presented in detail at [25]).

Global matrix method is employed in this study for dispersion analysis. The global matrix is formed by applying displacement and stress continuity boundary conditions at the interfaces. Assuming plane strain, motion confined to $x_{1}-x_{2}$ plane, a 3-layer system can be formed as seen in Figure 1, representing an acoustically thin waveguide immersed in a liquid. Wave motion in any layer can be imposed by suitable superposition of longitudinal $(L)$ and shear $(S)$ partial waves with $(+)$ or $(-)$ polarizations defined with respect to positive $x_{2}$ direction. At the semi-infinite half spaces (top and bottom), only the waves directed away from the interface and, in the intermediate solid waveguide layers, all four partial waves are considered. $S$ waves in the liquid (top and bottom) are kept for viscous fluid layers for a general case, although the liquids are assumed as being inviscid in this study (supporting only $L$-wave propagation).

The continuity of the displacements in $x_{1}$ and $x_{2}$ and the continuity of normal and shear stresses at the interfaces are considered to form the eigenvalue problem. Instead of layer matrices that are frequently used in similar problems, partial wave column vectors are used to form the global matrix. The problem for any scenario can be formed using proper arrangement of these partial wave vectors. For the threelayer system seen in Figure 1, the global matrix shown in (5) can be formed as shown below. Each row in the matrix corresponds to the continuity condition at the $i$ th interface (from top to the bottom) at $x_{2}=r_{i}$, and each element represents a partial wave column vector with 4 rows 
corresponding to the stresses and displacements of that partial wave as seen in Figure 1.

The wave vector $W$ shows the column order of displacements and stresses in (2). The four partial wave column vectors are given in (7) to (10), with their rows corresponding to the displacement and stress expressions given in matrix $W$. In the equations, $\rho$ is the density, $\lambda$ and $\mu$ are the Lame constants, $\omega$ is the angular speed, $\alpha$ is the bulk longitudinal, $\beta$ is the bulk shear velocity, and $k$ is the wave number (being equal to $\left.\omega / c_{p}\right)$. The liquid layers are also handled with 4-row column vectors, although they do not support shear motion or stress. As presented in [3], defining a very small shear velocity $(\sim 1 \mathrm{~m} / \mathrm{s})$ for the liquid layer is sufficient to define the system appropriately. Also, semi-infinite half space definition is utilized by defining very large the top and bottom layer thicknesses $(\sim 1000 \mathrm{~m})$.

$$
\left[\begin{array}{cccccccc}
-\left.L_{1,-}\right|_{r_{1}} & -S_{1,-} \mid r_{r_{1}} & \left.L_{2,+}\right|_{r_{1}} & \left.S_{2,+}\right|_{r_{1}} & L_{2,-} \mid r_{r_{1}} & \left.S_{2,-}\right|_{r_{1}} & 0 & 0 \\
0 & 0 & \left.L_{2,+}\right|_{r_{2}} & \left.S_{2,+}\right|_{r_{2}} & \left.L_{2,-}\right|_{r_{2}} & \left.S_{2,-}\right|_{r_{2}} & -\left.L_{3,+}\right|_{r_{2}} & -\left.S_{3,+}\right|_{r_{2}}
\end{array}\right]
$$$$
\cdot[A]=[0],
$$

$$
\begin{aligned}
& W=\left(\begin{array}{c}
u_{1} \\
u_{2} \\
\sigma_{22} \\
\sigma_{12}
\end{array}\right), \\
& L_{+}=\left(\begin{array}{c}
k g_{\alpha} \\
C_{\alpha} g_{\alpha} \\
i B \rho g_{\alpha} \\
i 2 \beta^{2} k C_{\alpha} \rho B g_{\alpha}
\end{array}\right) \text {, } \\
& S_{+}=\left(\begin{array}{c}
C_{\beta} g_{\beta} \\
-k g_{\beta} \\
-i 2 \beta^{2} k C_{\beta} \rho B g_{\beta} \\
i \rho B g_{\beta}
\end{array}\right) \text {, } \\
& L_{-}=\left(\begin{array}{c}
k g_{\alpha} \\
-C_{\alpha} g_{\alpha} \\
i B \rho g_{\alpha} \\
-i 2 \beta^{2} k C_{\alpha} \rho B g_{\alpha}
\end{array}\right) \text {, } \\
& S_{-}=\left(\begin{array}{c}
-C_{\beta} g_{\beta} \\
-k g_{\beta} \\
i 2 \beta^{2} k C_{\beta} \rho B g_{\beta} \\
i \rho B g_{\beta}
\end{array}\right) \text {, } \\
& \alpha=\sqrt{\frac{(\lambda+2 \mu)}{\rho}},
\end{aligned}
$$

$$
\begin{aligned}
\beta & =\sqrt{\frac{\mu}{\rho}}, \\
C_{\alpha} & =\sqrt{\frac{\omega^{2}}{\alpha^{2}}-k^{2}}, \\
C_{\beta} & =\sqrt{\frac{\omega^{2}}{\beta^{2}}-k^{2}}, \\
g_{\alpha} & =\exp \left(i C_{\alpha} x_{2}\right), \\
g_{\beta} & =\exp \left(i C_{\beta} x_{2}\right) .
\end{aligned}
$$

The large matrix given in (5) represents the global matrix $G$ for the problem, while the matrix $G$ multiplied by the amplitude matrix $A$ being equal to zero represents the eigenvalue problem. In order to have nontrivial solutions, the determinant of the matrix $G$ should be equal to zero. This equality gives the characteristic equation of the motion and the real roots of the equation correspond to the losslesspropagating waves for the given configuration and frequency. Phase velocity dispersion curves can be obtained by sweeping frequency or phase velocity, while keeping the other parameter constant. Since analytical evaluation of the characteristic equation is not possible, numerical methods are employed. Group velocity can also be defined for the propagating wave, which corresponds to the velocity of the travelling wavepacket, as guided waves are utilized in pulses for ultrasonic measurements thus travel in such way. Group velocity measurements are more frequently employed in dipstick sensor configuration, using two different immersion depths as presented in $[2,3]$. The group velocity can be calculated by using the phase velocity dispersion curve through the formula given below.

$$
c_{g}=c_{p}+c_{p} \frac{\partial c_{p}}{\partial \omega} .
$$

The numerical solution is performed in two steps. First, a coarse sweep of phase velocity is performed in steps of $1 \mathrm{~m} / \mathrm{s}$ with fixed frequency, while evaluating the absolute value of the determinant of the global matrix. All local minima are identified with this coarse sweep excluding local minimum points corresponding to the bulk wave velocities of the materials. A finer root finding is then performed around all the local minimum points using golden section bisection search. The method found especially effective for evaluating multiple quasi-Scholte roots of multilayer waveguides, compared with only-bisection search method [33].

\section{Dispersion Analysis}

Scholte waves show nondispersive behavior, when they propagate along the interface of two semi-infinite half spaces [3]. The wave velocity depends on the material properties of the interfacing materials (density, longitudinal and shear bulk wave of the solid, and density and speed of sound in the liquid), assuming perfectly elastic and isotropic materials. As mentioned previously, two distinct Scholte wave regions can 


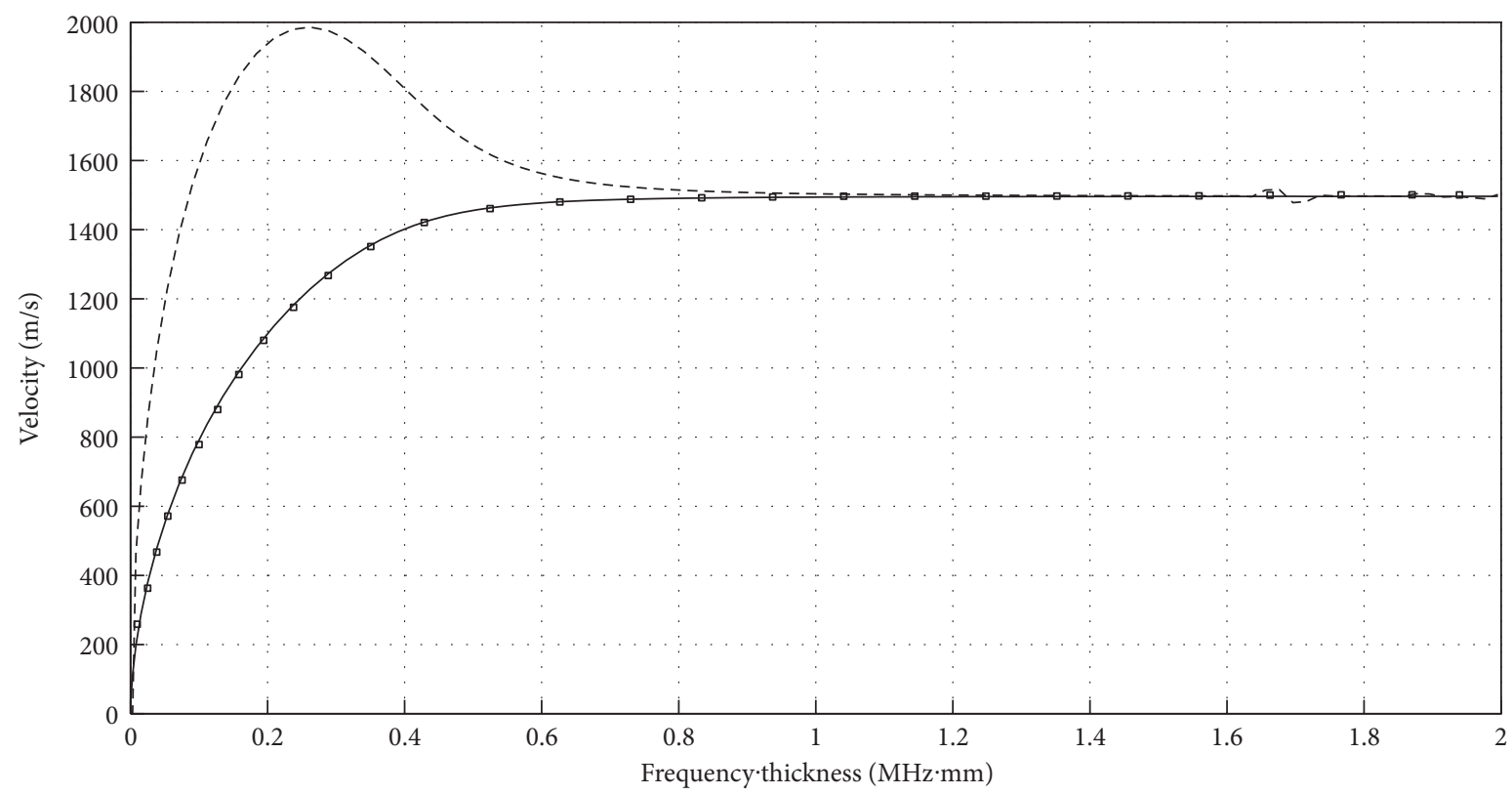

FiguRE 2: Dispersion curves for a $1 \mathrm{~mm}$ aluminum waveguide embedded in water: calculated phase velocity (solid), calculated group velocity (dashed), and experimentally verified phase velocity data points (square) digitized from Figure 1 of [3]. Material properties: $c_{L, \mathrm{al}}: 6320 \mathrm{~m} / \mathrm{s}$, $c_{S, \mathrm{al}}: 3130 \mathrm{~m} / \mathrm{s}, c_{L, w}: 1500 \mathrm{~m} / \mathrm{s}, \rho_{\mathrm{al}}: 2700 \mathrm{~kg} / \mathrm{m}^{3}$, and $\rho_{w}: 1000 \mathrm{~kg} / \mathrm{m}^{3}$.

be identified for the waves that propagate in a thin solid waveguide embedded (or dipped) in a liquid, for quasiScholte waves. In such case, Scholte wave phase velocity dispersion curve exhibits 0th order antisymmetric $A_{0}$ Lamb wave-like behavior, as seen in Figure 2 (solid line). In the dispersive region, starting from zero, the phase velocity increases by increasing frequency, asymptotically approaching the nondispersive value. The value of the nondispersive quasi-Scholte wave velocity is equal to the Scholte wave velocity for the same two materials in a semi-infinite interfacing half space configuration. Note that, for the actual $A_{0}$ Lamb wave mode, the phase velocity converges to the Rayleigh (surface) wave velocity. The curves presented in Figure 2 are representative for the configuration of $1 \mathrm{~mm}$ aluminum waveguide dipped in water. The $x$-axis of the graph is the thickness- (of the waveguide) frequency product for a more generalized representation, as the dispersion curve is the same if the thickness-frequency ( $t \cdot f)$ product is kept constant (in the 0 to $2 \mathrm{MHz} \cdot \mathrm{mm}$ range for Figure 2). Corresponding group velocity dispersion curve for the configuration is also presented in Figure 2 (dashed line). The resulting curves are validated with results from the literature, by experimentally verified phase velocity data points (square) digitized from Figure 1 of [3] and also presented in Figure 2. Note that group velocity is obtained through the dispersion curve for phase velocity through (13).

As stated previously, in this study, it is aimed to investigate sensitivity of quasi-Scholte wave to liquid properties. Thus, before proceeding forward, a detailed dispersion analysis is performed on the possible design space of liquid sensing using thin solid waveguides. Instead of exact dispersion curves for Scholte waves in a wide design space, a nondimensional approach has employed. In the author's previous studies, 2-D equal velocity curves (contours) were presented [33, 34]. Equal velocity curves, representing the ratio of the Scholte wave velocity to the longitudinal velocity of the liquid $\left(c_{\text {Scholte }} / c_{\text {liquid }}\right)$, have been obtained with respect to the density ratio $\left(\rho_{\text {solid }} / \rho_{\text {liquid }}\right)$ and longitudinal velocity ratio $\left(c_{L \text {,solid }} / c_{L \text {,liquid }}\right)$ of liquid to the solid waveguide; similar nondimensional Scholte wave velocity curves were presented in literature previously for the nondispersive Scholte waves [21]. In the analysis, it was seen that, unlike nondispersive waves, quasi-Scholte wave dispersion cannot be completely nondimensionalized. The curves were observed to be unaffected by the actual density but affected by the actual longitudinal velocity of liquid. The equal velocity contours are the same for the same density ratio, regardless of the actual values (the same curves for $\rho_{\text {solid }}=2000 \mathrm{~kg} / \mathrm{m}^{3}, \rho_{\text {liquid }}=1000 \mathrm{~kg} / \mathrm{m}^{3}$ or $\rho_{\text {solid }}=4000 \mathrm{~kg} / \mathrm{m}^{3}$, $\rho_{\text {liquid }}=2000 \mathrm{~kg} / \mathrm{m}^{3}$ ). However, it was seen that the actual values of the fluid longitudinal velocities affect the dispersion curves significantly, even though the ratio is kept constant $\left(c_{\text {solid }}=2000 \mathrm{~m} / \mathrm{s}, c_{L \text {,liquid }}=1000 \mathrm{~m} / \mathrm{s}\right.$ or $c_{\text {solid }}=4000 \mathrm{~m} / \mathrm{s}$, $c_{L \text {,liquid }}=2000 \mathrm{~m} / \mathrm{s}$ give different results). The 2-D nondimensional study with density and longitudinal velocity ratio has been expanded to a 3-D study with the addition of frequency (and for liquid longitudinal velocity) for a complete understanding of quasi-Scholte wave sensing design space.

A routine is developed in MATLAB, following the analytical model presented in the previous section for the analysis. The parametrized design space is listed in Table 1. Solutions of all possible combinations of the 4 parameters in listed steps have been calculated, and the following figures have 
TABLE 1: Input parameters for dispersion analysis.

\begin{tabular}{|c|c|c|c|c|}
\hline & Name & Symbol & Range & Step \\
\hline \multirow{4}{*}{ Input parameters } & Density ratio & $\frac{\rho_{\text {solid }}}{\rho_{\text {liquid }}}$ & $0.5-5$ & 0.5 \\
\hline & Long. vel. ratio & $\frac{c_{L, \text { solid }}}{c_{L, \text { liquid }}}$ & $0.5-5$ & 0.5 \\
\hline & Frequency.thickness (MHz) & $f \cdot t$ & $0.1-1.5$ & 0.1 \\
\hline & Fluid long. vel. $(\mathrm{m} / \mathrm{s})$ & $c_{L, \text { liquid }}$ & $500-2500$ & 100 \\
\hline
\end{tabular}

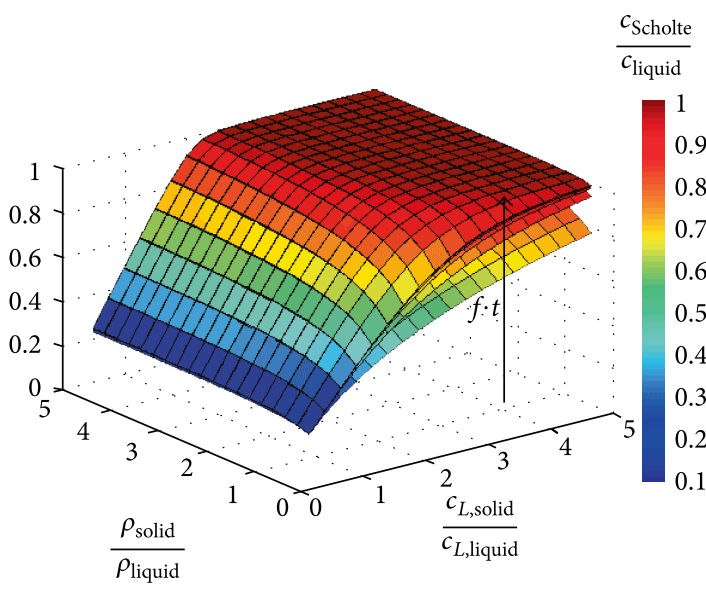

(a)

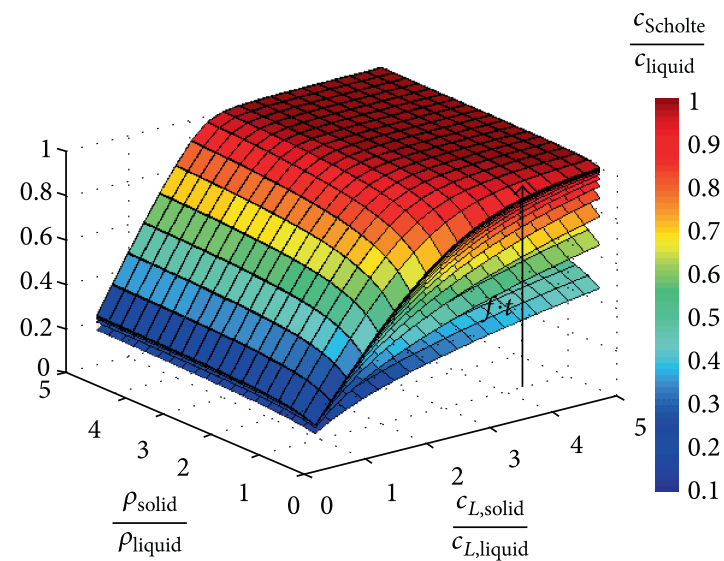

(b)

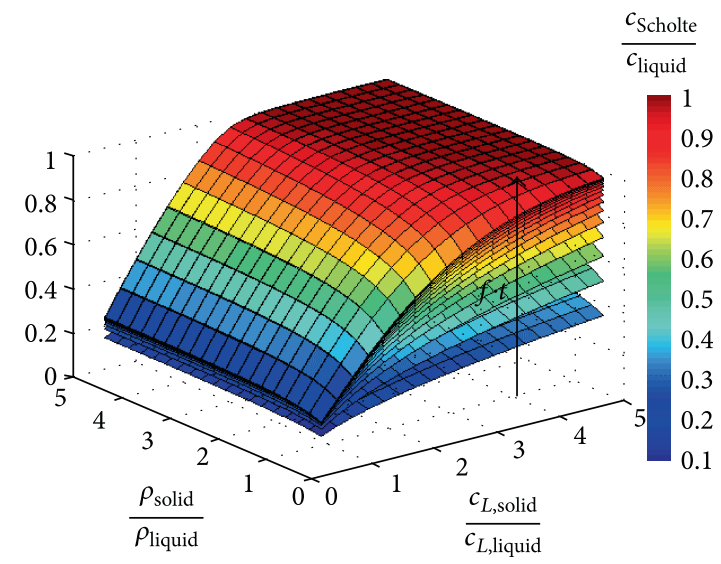

(c)

FIGURE 3: Nondimensional Scholte velocity dispersion surfaces $\left(c_{\text {Scholte }} / c_{\text {liquid }}\right)$ for $c_{L, \text { liquid }}$ of (a) $500 \mathrm{~m} / \mathrm{s}$, (b) $1500 \mathrm{~m} / \mathrm{s}$, and (c) $2500 \mathrm{~m} / \mathrm{s}$ ( $x$-axis: $c_{L, \text { solid }} / c_{L, \text { liquid }}, y$-axis: $\rho_{\text {solid }} / \rho_{\text {liquid }}$, arrow pointing increasing frequency thickness product direction).

been prepared for illustration. Since the data is large, it is not possible to illustrate all the findings, thus only the representative results have been illustrated.

Representative nondimensional Scholte wave surfaces are presented in Figure 3. The colors represent the $c_{\text {Scholte }} / c_{\text {liquid }}$ ratio (also presented in $z$-axis) as a function of $c_{L, \text { solid }} / c_{L, \text { liquid }}$ ratio $\left(x\right.$-axis) and $\rho_{\text {solid }} / \rho_{\text {liquid }}$ ratio for increasing frequency towards the arrow direction for $c_{L, \text { liquid }}$ of $500 \mathrm{~m} / \mathrm{s}$ Figure $3(\mathrm{a}), 1500 \mathrm{~m} / \mathrm{s}$ Figure $3(\mathrm{~b})$, and $2500 \mathrm{~m} / \mathrm{s}$ Figure 3(c).
As a general trend, it can be depicted from the figures that Scholte wave velocity is less sensitive to the changes in density ratio, compared to the changes in longitudinal velocity ratio. For the low end of the fluid longitudinal velocity, the surfaces are pretty much identical after $0.2 \mathrm{MHz} \cdot \mathrm{mm}$. The surfaces move away from each other $\left(c_{\text {Scholte }} / c_{\text {liquid }}\right.$ changes significantly) for changing frequency with increasing $c_{L, \text { liquid }}$. Note that the upper most surfaces are identical for Figures 3(a)-3(c), which correspond to the nondispersive 
TABLE 2: Liquid properties used for changing fluid longitudinal velocity and density in Figure 7 . The value on the $y$-axis equal to the fluid longutidunal velocity $\left(c_{L, \text { liquid }}\right)$.

\begin{tabular}{|c|c|c|c|c|c|c|c|c|c|c|c|c|c|}
\hline$c_{L, \text { liquid }}(\mathrm{m} / \mathrm{s})$ & 1200 & 1300 & 1400 & 1500 & 1600 & 1700 & 1800 & 1900 & 2000 & 2100 & 2200 & 2300 & 2400 \\
\hline$\rho_{\text {liquid }}\left(\mathrm{kg} / \mathrm{m}^{3}\right)$ & 800 & 900 & 1000 & 1100 & 1200 & 1300 & 1400 & 1500 & 1600 & 1700 & 1800 & 1900 & 2000 \\
\hline
\end{tabular}

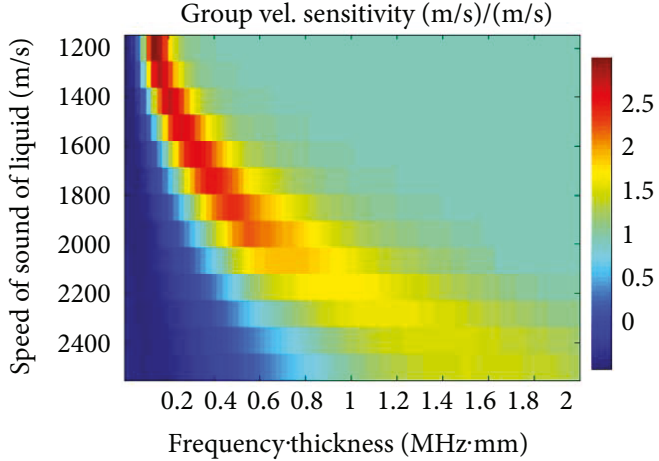

(a)

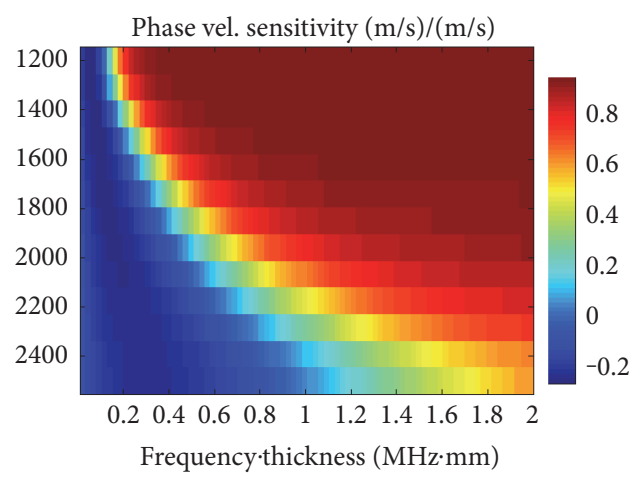

(b)

FIGURE 4: Sensitivity to changing liquid longitudinal velocity for aluminum waveguide dipped in liquid: (a) group velocity sensitivity and (b) phase velocity sensitivity. Material properties of aluminum same as given in Figure 2 caption, $\rho_{\text {liquid }}=1000 \mathrm{~kg} / \mathrm{m}^{3}$ (constant).

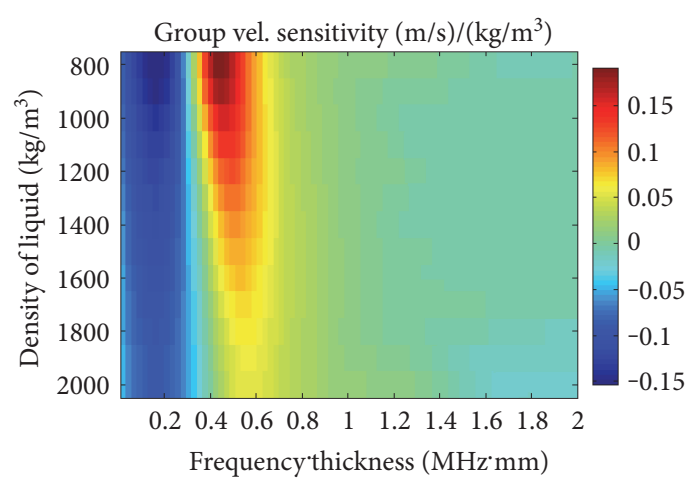

(a)

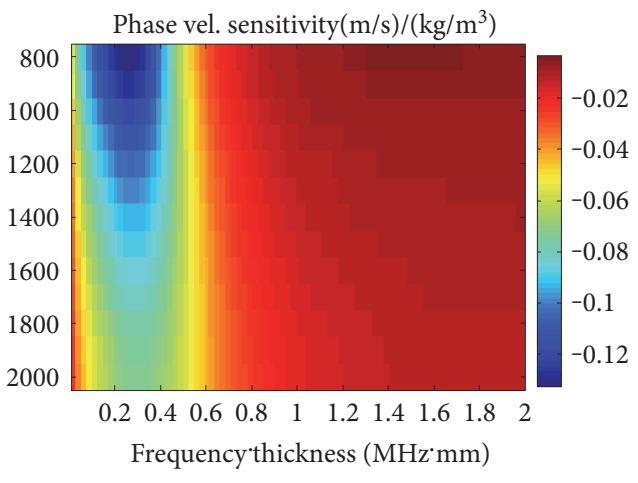

(b)

FIGURE 5: Sensitivity to changing liquid density for aluminum waveguide dipped in liquid: (a) group velocity sensitivity and (b) phase velocity sensitivity. Material properties of aluminum same as given in Figure 2 caption, $c_{L, \text { liquid }}=1500 \mathrm{~m} / \mathrm{s}$ (constant).

equal velocity surface. Ensuring frequency is high enough; the surface is perfectly nondimensional, that is, it does not depend on absolute value of any parameter involved.

\section{Sensitivity Analysis}

Sensitivity analysis for (water-like) liquid sensing utilizing thin solid waveguides is performed analytically using the developed dispersion routine. A dimensional approach is rather chosen, by means of both sensitivity and the material properties in order to make a comparison in absolute values of the quantities. The sensitivity is defined as the ratio of the change in sensed quantity $\left(c_{p, g}\right.$ : Scholte wave phase or group velocity) to the varying parameter ( $x$ : density, longitudinal velocity, or both) with a first order approximation as seen in (14). A range of 800 to $2000 \mathrm{~kg} / \mathrm{m}^{3}$ is chosen for the density, and 1200 to $2400 \mathrm{~m} / \mathrm{s}$ is chosen for the fluid longitudinal velocity, covering a design space typical to industrial water-like solutions, mixtures in beverage industry and similar.

$$
\text { Sensitivity }_{p, g}=\frac{\partial c_{p, g}}{\partial x} \approx \frac{\Delta c_{p, g}}{\Delta x}
$$

The sensitivity is analyzed in three cases with respect to (i) changing fluid longitudinal velocity, while fluid density is kept constant, (ii) changing fluid density, while fluid longitudinal velocity is kept constant, and (iii) changing fluid density and longitudinal velocity with the same step size (given in detail at Table 2); and the results are presented in Figures 4, 5, and 6, respectively, for the three cases. The Scholte wave sensitivity to changing fluid longitudinal velocity is found to be an order of magnitude higher compared to 


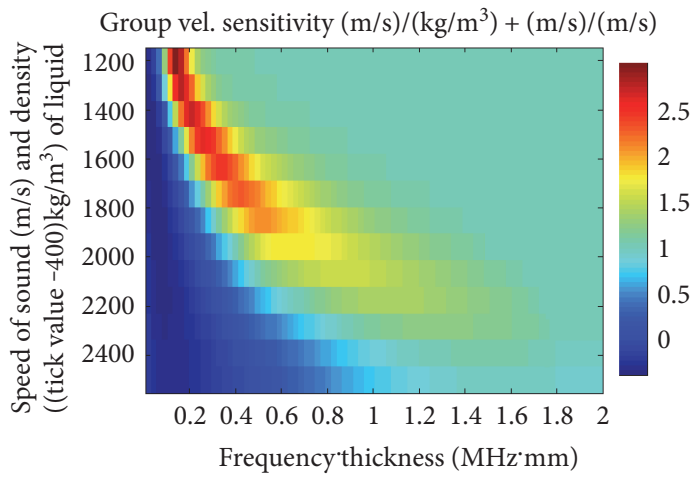

(a)

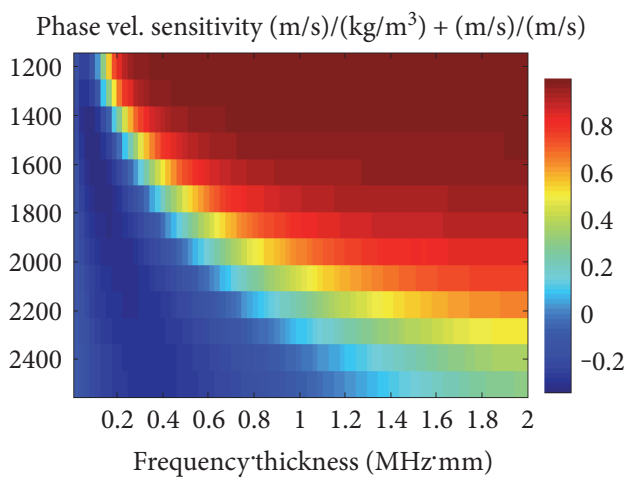

(b)

FIGURE 6: Sensitivity to changing liquid longitudinal velocity and density for aluminum waveguide dipped in liquid: (a) group velocity sensitivity and (b) phase velocity sensitivity. Material properties of aluminum same as given in Figure 2 caption; the liquid properties used are listed in Table 2.

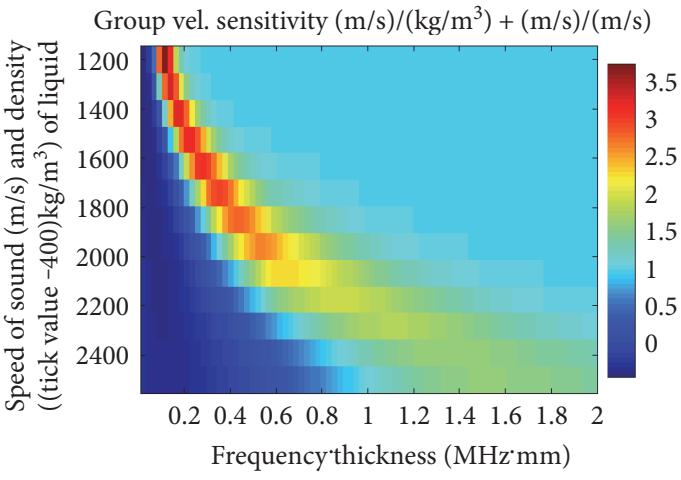

(a)

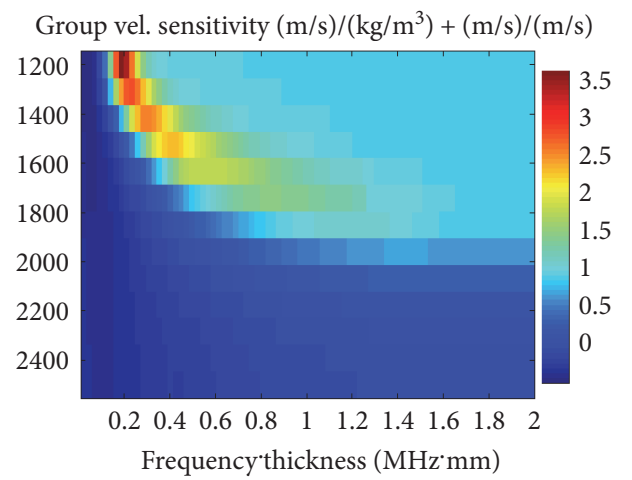

(b)

FIGURE 7: Group velocity sensitivity to changing liquid longitudinal velocity and density for (a) steel, (b) brass waveguide. The liquid properties used is listed in Table 2. Waveguide material properties of steel: $c_{L, \mathrm{st}}=5950 \mathrm{~m} / \mathrm{s}, c_{S, \mathrm{st}}=3150 \mathrm{~m} / \mathrm{s}$, and $\rho_{\mathrm{st}}=7700 \mathrm{~kg} / \mathrm{m}^{3} ; \mathrm{brass}$ : $c_{L, \mathrm{br}}=4700 \mathrm{~m} / \mathrm{s}, c_{S, \mathrm{br}}=2110 \mathrm{~m} / \mathrm{s}$, and $\rho_{\mathrm{br}}=8600 \mathrm{~kg} / \mathrm{m}^{3}$.

changing fluid density both for group and phase velocity sensitivities as seen in Figures 4 and 5. Since the sensitivity to fluid longitudinal velocity is higher, this property also dominates the sensitivity when both density and velocity are swept (Figure 6), leading to similar sensitivity plots for changing velocity only and changing velocity and density (Figures 4 and 6). The red regions in all group velocity sensitivity plots (Figures 4(a), 5(a), and 6(a)) correspond to the optimal sensor configuration showing the highest sensitivity. In Figures 4 and 6, this optimal region extends into a wide range by means of both frequency thickness product and changing material properties. Phase velocity sensitivities are given as reference, although they are not frequently employed in dipstick sensing, since group velocity measurements are easier to obtain [3].

It is also essential to investigate the effect of the waveguide material on sensitivity. Steel and brass, which are also frequently used for aqueous applications, are chosen for a brief study on the effect of sensitivity in addition to aluminum. The results are presented in Figure 7, only in group velocity sensitivity to changing longitudinal velocity and density (similar to Figure 6(a)) for steel Figure 7(a) and for brass Figure 7(b). The material properties of the steel and brass are given in the caption of Figure 7, and aluminum is already presented in Figure 2 caption. They provide a good insight for material selection, since steel has acoustic velocities similar to aluminum, but a higher density $\left(7700 \mathrm{~kg} / \mathrm{m}^{3}\right)$, and brass has a density close to steel $\left(8600 \mathrm{~kg} / \mathrm{m}^{3}\right)$ but lower acoustic velocities $\left(c_{L}=4700 \mathrm{~m} / \mathrm{s}\right.$ and $\left.c_{S}=2110 \mathrm{~m} / \mathrm{s}\right)$. As can be depicted from Figure 7(a), increased density of the waveguide not only increased the sensitivity to even higher values but also further extended the high sensitivity range.

The extended sensitivity range is especially essential for flexible design of sensing systems in which high-frequency transducers and/or waveguides with higher thicknesses can be employed. For the brass waveguide case (Figure 7(b)), peak sensitivity similar to steel is obtained; however, the high sensitivity range is limited to low frequency thickness and fluid longitudinal velocity values. Comparing Figures 6(a), $7(\mathrm{a})$, and 7(b), it can be concluded that higher waveguide material density leads to higher sensitivities, and higher waveguide acoustic velocities lead to an extended effective sensing range, in the investigated design space. 


\section{Discussion and Conclusions}

In this manuscript, the study on quasi-Scholte-guided wave dispersion and sensitivities of these waves to changes in fluid density and speed of sound in a dipstick sensor configurations are presented. An analytical model is used to model the multilayer system and trace dispersion curves. A twostep numerical method is used to generate dispersion curves and nondimensional Scholte wave velocity surfaces as a function of material properties of the waveguide and the fluid. The sensitivity of quasi-Scholte waves is then analytically analyzed for typical metal waveguide materials (aluminum, steel, and brass) and for liquids with material properties representing industrial water-like solutions and mixtures in beverage industry.

Through the nondimensional dispersion analysis, it is found that Scholte wave velocity is more sensitive to changing longitudinal velocity ratio, compared to the changing density ratio. It is also seen that the nondimensional Scholte wave surface profile is affected by the absolute value of the liquid longitudinal velocity. The surfaces are observed to move away from each other for changing frequency with increasing liquid longitudinal velocity. Ensuring frequency is high enough; the surfaces are found to converge to a perfectly nondimensional surface, that is, it does not depend on absolute value of any parameter involved.

The sensitivity is analyzed in three cases with respect to (i) changing fluid longitudinal velocity, while fluid density is kept constant, (ii) changing fluid density, while fluid longitudinal velocity is kept constant, and (iii) changing fluid density and longitudinal velocity. The Scholte wave sensitivity to changing fluid longitudinal velocity is calculated to be an order of magnitude higher compared to changing fluid density both for group and phase velocity sensitivities. The sensitivity to fluid longitudinal velocity is similar to the sensitivity profile when both density and velocity are swept in same increments. Optimal sensor configurations showing high sensitivity and wide range are identified. The effect of the waveguide selection on sensitivity is also evaluated and it is estimated that increasing waveguide material density increases the sensitivity and extends the range of effective sensing.

The resulting sensitivity plots can be employed to develop highly sensitive quasi-Scholte wave sensors depending on the application for different sensing purposes. The results of the study have shown that high sensitivity regions should be traced for a specific application, if high sensitivity is sought. Most of the time, dipstick ultrasonic-guided sensing mechanisms are limited by the availability of the transducers and the waveguides, which are usually mass produced at specific frequencies (for the transducers) and thickness and material properties (for the waveguides). Even though a sensitivity peak is identified, it might not be feasible to obtain such configuration, because of the high cost and limited availability. The nondimensional sensitivity plots presented in the study present a guide for material and transducer selection by identifying the nondimensionalized high sensitivity regions. The transducer and waveguide properties can be altered according to the application (target fluid), ensuring the sensing configuration has the highest possible sensitivity. Furthermore, isolation of a target sensing quantity from other changing physical quantity is also an important issue. In acoustic fluid sensing, density change is usually accompanied by changes in fluid longitudinal velocity, which complicates the sensing method. Also by selection of transducer and waveguide, either density or fluid longitudinal velocities can be traced independently from the other. By clearly understanding the ranges and configurations for different sensitivity regions such as maximum, zero, or negative sensitivity, precision multimode or multiquantity sensors could be developed.

\section{Conflicts of Interest}

The author declares that there is no conflict of interest regarding the publication of this paper.

\section{Acknowledgments}

This work is supported by The Scientific and Technological Research Council of Turkey (TUBITAK) through Grant $114 \mathrm{C} 102$ and by Izmir Institute of Technology BAP Grant 2015IYTE26. The author would also like to thank ASELSAN AS for encouragement and support for this publication.

\section{References}

[1] G. F. Dye and J. S. McSpadden, "Ultrasonic linear meter sensor for positive displacement meter," Google Patents, 1990.

[2] F. B. Cegla, "Ultrasonic waveguide sensors for fluid characterisation and remote sensing," in Department of Mechanical Engineering, p. 248, Imperial College London, London, UK, 2006.

[3] F. B. Cegla, P. Cawley, and M. J. S. Lowe, "Material property measurement using the quasi-Scholte mode-a waveguide sensor," The Journal of the Acoustical Society of America, vol. 117, no. 3, pp. 1098-1107, 2005.

[4] Z. Fan, M. J. S. Lowe, M. Castaings, and C. Bacon, "Torsional waves propagation along a waveguide of arbitrary cross section immersed in a perfect fluid," The Journal of the Acoustical Society of America, vol. 124, no. 4, pp. 2002-2010, 2008.

[5] Y. Ai and R. A. Lange, "Theoretical analyses and numerical simulations of the torsional mode for two acoustic viscometers with preliminary experimental tests," IEEE Transactions on Ultrasonics, Ferroelectrics, and Frequency Control, vol. 55, no. 3, pp. 648-658, 2008.

[6] R. Kazys, L. Mazeika, R. Sliteris, and R. Raisutis, "Measurement of viscosity of highly viscous non-Newtonian fluids by means of ultrasonic guided waves," Ultrasonics, vol. 54, no. 4, pp. 1104-1112, 2014.

[7] J. Ma, M. J. S. Lowe, and F. Simonetti, "Measurement of the properties of fluids inside pipes using guided longitudinal waves," IEEE Transactions on Ultrasonics, Ferroelectrics, and Frequency Control, vol. 54, no. 3, pp. 647-658, 2007.

[8] F. Padilla, M. de Billy, and G. Quentin, "Theoretical and experimental studies of surface waves on solid-fluid interfaces when the value of the fluid sound velocity is located between the shear and the longitudinal ones in the solid," The Journal of the Acoustical Society of America, vol. 106, no. 2, pp. 666-673, 1999. 
[9] C. L. Shepard, B. J. Burghard, M. A. Friesel et al., "Measurements of density and viscosity of one- and two-phase fluids with torsional waveguides," IEEE Transactions on Ultrasonics Ferroelectrics, and Frequency Control, vol. 46, no. 3, pp. 536548, 1999.

[10] N. N. Subhash and K. Balasubramaniam, "Fluid level sensing using ultrasonic waveguides," Insight - Non-Destructive Testing and Condition Monitoring, vol. 56, no. 11, pp. 607-612, 2014.

[11] A. E. Takiy, S. C. G. Granja, R. T. Higuti et al., "Theoretical analysis and experimental validation of the scholte wave propagation in immersed plates for the characterization of viscous fluids," in 2013 IEEE International Ultrasonics Symposium (IUS), Prague, Czech Republic, July 2013.

[12] T. Vogt, M. Lowe, and P. Cawley, "Cure monitoring using ultrasonic guided waves in wires," The Journal of the Acoustical Society of America, vol. 114, no. 3, pp. 1303-1313, 2003.

[13] T. Vogt, M. J. S. Lowe, and P. Cawley, "Ultrasonic waveguide techniques for the measurement of material properties," AIP Conference Proceedings, vol. 615, no. 1, pp. 1742-1749, 2002.

[14] S. Periyannan and K. Balasubramaniam, "Moduli determination at different temperatures by an ultrasonic waveguide method," Experimental Mechanics, vol. 56, no. 7, pp. 12571270, 2016.

[15] W. Voss, "Ultrasonic filling level sensor," Google Patents, 2007.

[16] S. Periyannan, P. Rajagopal, and K. Balasubramaniam, "Robust ultrasonic waveguide based distributed temperature sensing," Physics Procedia, vol. 70, Supplement C, pp. 514518, 2015.

[17] S. Periyannan, P. Rajagopal, and K. Balasubramaniam, "Torsional mode ultrasonic helical waveguide sensor for reconfigurable temperature measurement," AIP Advances, vol. 6, no. 6, article 065116, 2016.

[18] Z. Tung-Sheng, W. Sun-Li, and T. Wen-Yuan, "Spontaneous and highly accurate ultrasonic temperature measurement system for air conditioner in automobiles," Journal of Scientific and Industrial Research, vol. 68, p. 44, 2009.

[19] A. Afaneh, S. Alzebda, V. Ivchenko, and A. N. Kalashnikov, "Ultrasonic measurements of temperature in aqueous solutions: why and how," Physics Research International, vol. 2011, Article ID 156396, 10 pages, 2011.

[20] P. Rizzo, J.-G. Han, and X.-L. Ni, "Structural health monitoring of immersed structures by means of guided ultrasonic waves," Journal of Intelligent Material Systems and Structures, vol. 21, no. 14, pp. 1397-1407, 2010.

[21] G. D. Meegan, M. F. Hamilton, Y. A. Il'inskii, and E. A. Zabolotskaya, "Nonlinear Stoneley and Scholte waves," The Journal of the Acoustical Society of America, vol. 106, no. 4, pp. 1712-1723, 1999.

[22] F. B. Cegla, P. Cawley, and M. J. S. Lowe, "Fluid bulk velocity and attenuation measurements in non-Newtonian liquids using a dipstick sensor," Measurement Science and Technology, vol. 17, no. 2, pp. 264-274, 2006.

[23] S. Nasr, J. Duclos, and M. Leduc, "Scholte wave characterization and its decay for various materials," The Journal of the Acoustical Society of America, vol. 87, no. 2, pp. 507-512, 1990.

[24] O. Onen and Y. C. Uz, "Investigation of Scholte and Stoneley waves in multi-layered systems," Physics Procedia, vol. 70, pp. 217-221, 2015.
[25] M. J. S. Lowe, "Matrix techniques for modeling ultrasonic waves in multilayered media," IEEE Transactions on Ultrasonics Ferroelectrics and Frequency Control, vol. 42, no. 4, pp. 525-542, 1995.

[26] W. T. Thomson, "Transmission of elastic waves through a stratified solid medium," Journal of Applied Physics, vol. 21, no. 2, pp. 89-93, 1950.

[27] B. A. Auld, Acoustic Fields and Waves in Solids, R.E. Krieger, Malabar, FL, USA, 2nd edition, 1990.

[28] J. W. Dunkin, "Computation of modal solutions in layered, elastic media at high frequencies," Bulletin of the Seismological Society of America, vol. 55, no. 2, pp. 335-358, 1965.

[29] H. Schmidt and G. Tango, "Efficient global matrix approach to the computation of synthetic seismograms," Geophysical Journal International, vol. 84, no. 2, pp. 331-359, 1986.

[30] K. Balasubramaniam, "On a numerical truncation approximation algorithm for transfer matrix method," The Journal of the Acoustical Society of America, vol. 107, no. 2, pp. 1053-1056, 2000.

[31] A. Pilarski, J. L. Rose, and K. Balasubramaniam, “The angular and frequency characteristics of reflectivity from a solid layer embedded between two solids with imperfect boundary conditions," The Journal of the Acoustical Society of America, vol. 87, no. 2, pp. 532-542, 1990.

[32] L. Wang and S. I. Rokhlin, "Stable reformulation of transfer matrix method for wave propagation in layered anisotropic media," Ultrasonics, vol. 39, no. 6, pp. 413-424, 2001.

[33] Y. Uz, O. Bostan, G. Ç. Akyol, and O. Önen, “An ultrasonic waveguide sensor for monitoring alcohol concentration in water-alcohol mixtures," in Proceedings of the 22nd International Congress on Acoustics, Buenos Aires, Argentina, September 2016.

[34] J. Rautenberg and M. Münch, "Concentration measurement in bubbly liquids - a matter of times," Physics Procedia, vol. 70, pp. 147-150, 2015. 


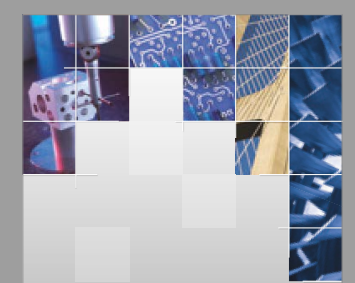

\section{Enfincering}
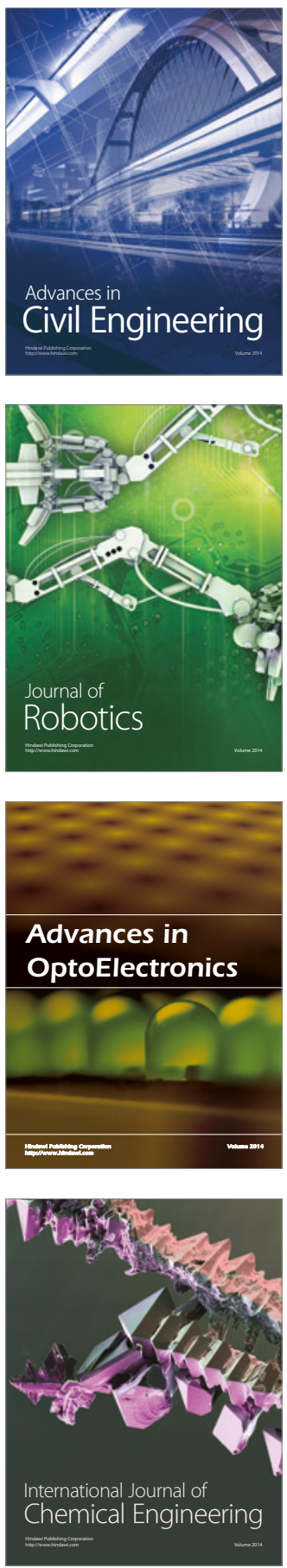

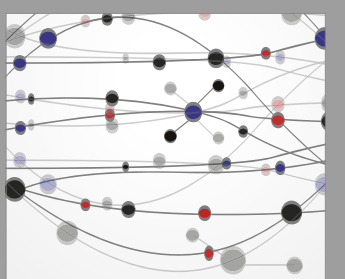

The Scientific World Journal

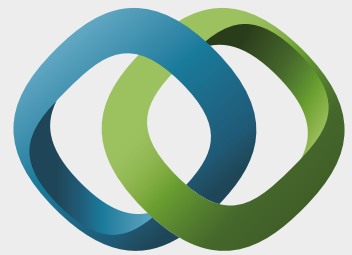

\section{Hindawi}

Submit your manuscripts at

https://www.hindawi.com
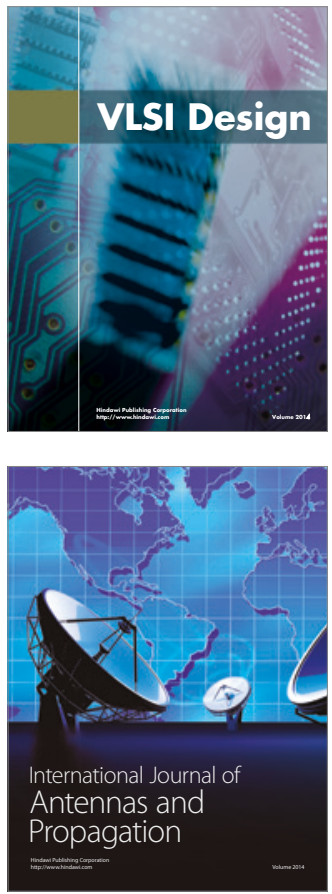

\section{Rotating}

Machinery
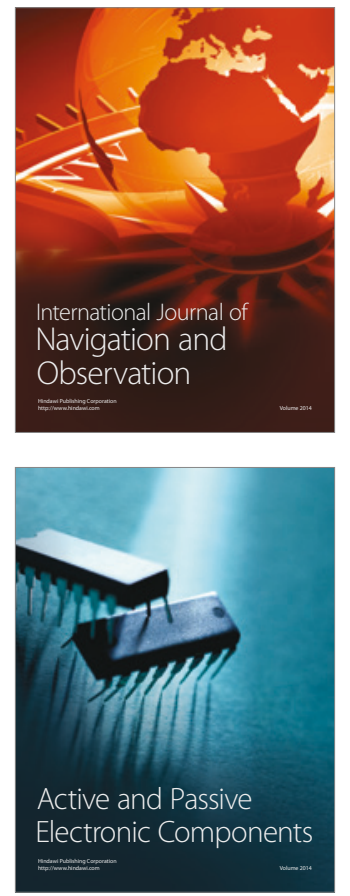
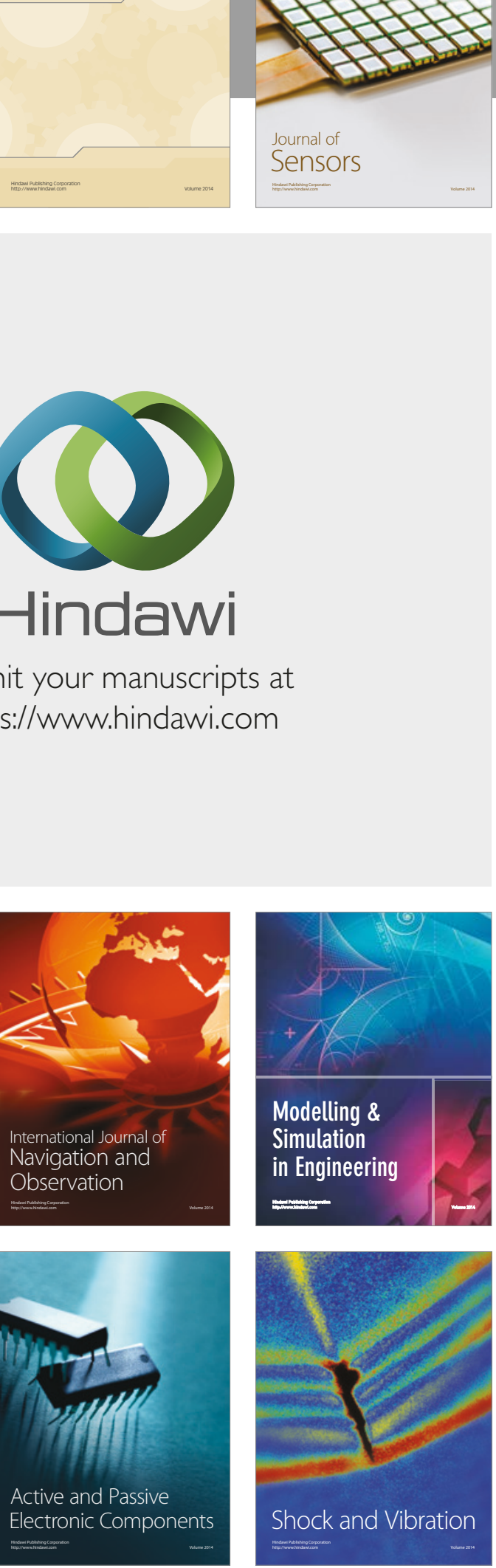
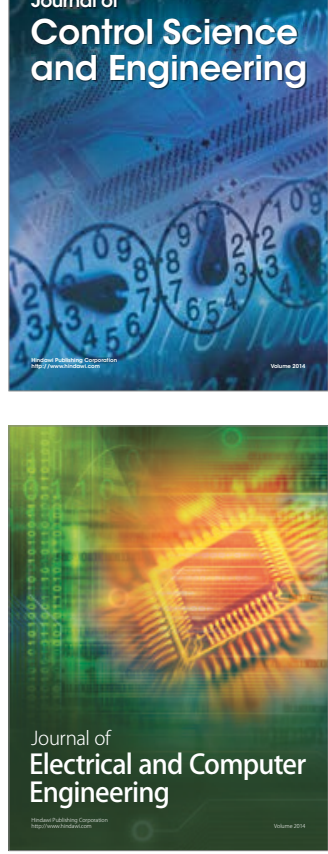

Distributed

Journal of

Control Science

and Engineering
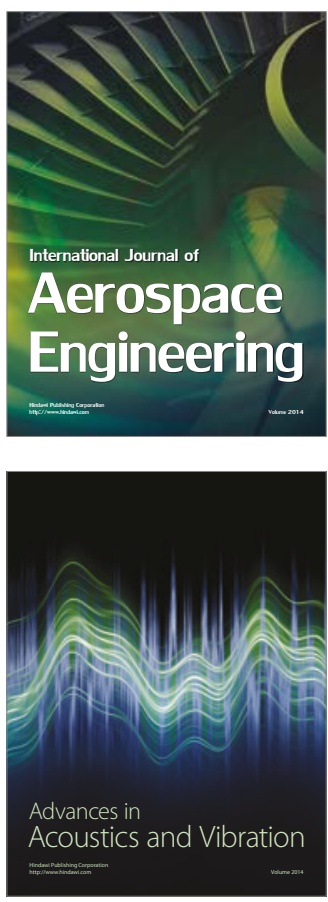

Sensor Networks 